\title{
El problema de la investigación sobre los niños y la televisión ${ }^{1}$
}

\author{
Roberto Farné \\ Profesor ordinario de Didáctica General. Universidad de Bolonia.
}

Resumen:

La televisión ha sido hasta ahora el medio más estudiado por las Ciencias Sociales y de la Educación, a través de una enorme cantidad de investigaciones empíricas. Medio siglo de investigación sobre la relación entre los niños y la televisión nos llevan a dos consideraciones: la primera es que ningún resultado ha alcanzado los términos de "verdad científica", o sea, que los efectos del medio sobre el comportamiento y la personalidad infantiles no están objetivamente definidos. Todo parece depender de las "condiciones" del sujeto (edad, ambiente, educación, etc.). La segunda consideración es que si se excluyen algunos casos de programas televisivos para la infancia (el más famoso es Barrio Sésamo), las investigaciones sobre la televisión y los niños no han tenido ninguna influencia sobre las emisiones televisivas, sobre la selección de ideas y productos. A partir de estas consideraciones, y de algunas investigaciones históricamente significativas, el artículo trata de poner en claro el problema epistemológico de este campo de investigación, entre la "verdad" y el "sentido común".

Palabras clave:

Televisión, infancia, investigación, educación

Abstract:

Television has been the most studied medium by the social and educational sciences so far, through a huge number of empirical researches. Half a century of research on the relationship between children and television leads us to two considerations: the first one is that no outcome has been achieved in terms of "Scientific Truth"; the effects of this medium on children's behaviour and personality are not definable from an objective point of view. All seems to depend on the personal "conditions" of the individual (age, environment, education etc...). The second one is that, excluding some cases of children's TV programs, like the most renowned Sesame Street, the researches on TV and childhood had no influence on the ideas and the production ways of TV broadcasters. Starting from these considerations, and from some significant researches, this article tries to focus the epistemological problem of this field of research, between "truth" and "common sense".

\section{Keywords:}

\section{Television, childhood, research}

\footnotetext{
1 Traducción a cargo de Ignacio Blanco Alfonso del original italiano Il problema della ricerca su bambini e televisione.
} 


\section{Introducción}

"Para ciertos niños, en ciertas condiciones, una cierta televisión es nociva. Para otros niños en las mismas condiciones, o para los mismos niños en otras condiciones, puede ser útil. Para la mayor parte de los niños, en la mayor parte de las condiciones, la mayor parte de la televisión no es, probablemente, ni particularmente nociva ni particularmente útil".

Con esta frase comienza la introducción de un voluminoso trabajo de investigación (casi 400 páginas en la edición italiana) publicado en 1961 con el título Television in the Lives of Our Children y cuyos autores son Wilbur Schramm, Jack Lyle y Edwin Parker. Resultado de once estudios relacionados entre ellos y llevados a cabo entre Estados Unidos y Canadá en el trienio 1958-1960, implicando algunos millares de sujetos agrupados sobre la base de rigurosos criterios de selección, esta investigación ha sido durante muchos años un punto de referencia para quien quería indagar en este campo.

La frase que he referido y con la que se abre el libro puede parecer asombrosa y desconsoladora al mismo tiempo, casi una provocación. Los mismos autores son conscientes de ello pues afirman, acto seguido, que más allá del tono demasiado prudente, lleno de ambigüedad, simple en sus consideraciones, el tema que han afrontado es muy complejo. Tan complejo que resulta imposible llegar a demostrar unas tesis claras y distintas sobre los efectos positivos o negativos del medio y de sus programas sobre determinadas tipologías de público. Normalmente es esto lo que se espera de las investigaciones sobre la televisión: saber lo que beneficia y lo que perjudica, reglas de comportamiento, juicios precisos, datos estadísticos fidedignos de los cuales recabar "datos sobre la verdad".

Schramm, Lyle y Parker desarrollan una operación singular para los investigadores: en la introducción [de la investigación] no plantean la pregunta y las hipótesis que justifican todo el trabajo de investigación, pero sí anticipan con suma síntesis la conclusión. El término "efecto" sobre el que siempre se ha concentrado gran parte de la atención científica sobre el problema de la relación entre la televisión y la infancia, afirman los autores, se revela en realidad desorientador “porque parece sugerir que la televisión 'hace algo' a los niños (...). De esta forma, los niños aparecen relativamente inertes y la televisión relativamente activa. Los niños sentados delante de la pantalla como víctimas, y la televisión está allí dispuesta a devorarlos. Nada más lejos de la realidad. En este estudio los más activos son los niños; son ellos quienes usan la televisión y no viceversa".

El fundamento epistemológico de tipo behaviorista sobre el que se han fundado muchísimas investigaciones en este campo, viene sustituido con otro sistémico, basado en la complejidad de las redes relacionales

$14 \mid n^{\circ} 7$ | doxa.comunicación 
entre sujeto y contexto socio-cultural, sobre la centralidad de la experiencia educativa. El capítulo final se cierra con la afirmación introductoria: "Parece claro que para comprender el impacto y el efecto de la televisión sobre los niños debemos, antes que nada, abandonar el concepto no realista de lo que la televisión "hace a los niños' y sustituirlo por aquel de lo que los niños hacen con la televisión". Una afirmación, esta última, que en tiempos recientes ha aparecido en muchos estudios e investigaciones cuyos autores, quizá, no saben o han olvidado que 50 años atrás alguien ya la había dicho, sosteniéndola con un robusto aparato científico.

La investigación de Schramm, Lyle y Parcker no se termina con las respuestas, como obviamente se podría pensar, sino con un conjunto de preguntas seguidas por amplias argumentaciones dirigidas a interlocutores específicos. Veamos algunas. En el epígrafe Preguntas a los responsables de las redes y de las estaciones televisivas, los autores preguntan: “ $¿ E s$ posible producir programas que lleguen a atraer a los niños sin recurrir a altas dosis de violencia y de tensión?”Y más adelante: “¿Es posible adecuar mejor a las necesidades de los niños la imagen de la vida adulta que ahora representa la televisión comercial?” El sentido de estas preguntas y las argumentaciones con que vienen acompañadas no tienen un significado simplemente retórico, sino que están basadas en los datos de la propia investigación. Son las preguntas los auténticos "datos de verdad" de la investigación.

En el epígrafe Preguntas para los padres se plantea: "Los padres que temen la influencia de la televisión sobre sus hijos, ¿̇hacen cuanto está en sus manos para darles un sentido de seguridad, de amor y de 'pertenencia'?” Otra pregunta es: "Los padres que se preocupan del bajo nivel intelectual del contenido de los programas televisivos, ihacen todo lo posible por encaminar a sus hijos hacia alguna experiencia televisiva más estimulante?" No faltan, a continuación, un epígrafe con las Preguntas para las escuelas (¿se hace todo lo posible por tener en cuenta la televisión en la experiencia cultural de los niños?); uno con las Preguntas para la autoridad gubernamental (¿hacen todo lo posible para facilitar y animar la producción de buenos programas destinados a los niños?), y finalmente un epígrafe con las Preguntas para los investigadores. Aquí los autores plantean el problema de qué modelo de investigación sea el más adecuado para afrontar la complejidad de un tema como éste.

Afirman que existen tres tipologías fundamentales de investigaciones: una a gran escala, que ellos mismos han utilizado para desarrollar su investigación; el experimento de laboratorio, que tiene características más específicas e implica un número limitado de sujetos; y tercer tipo de estudio, intensivo y a largo plazo, llevado a cabo sobre un número relativamente pequeño de niños. Se trataría en este caso de conocer a fondo los sujetos implicados para poder comprender los efectos de la experiencia televisiva a través de observaciones y experimentos en un periodo de tiempo relativamente largo. 
A medio siglo de distancia de la investigación de Schramm, Lyle y Parcker, ¿no son éstas (junto a otras) las preguntas de fondo que todavía hoy se plantean en los estudios e investigaciones sobre la relación entre los niños y la televisión?

Este salto atrás en la investigación científica no está motivado por una actitud de nostalgia del pasado o de escepticismo sobre el presente, sino del hecho de que si perdemos de vista la perspectiva histórica de la investigación en este campo, nos arriesgamos, por una parte, a pensar que los resultados actuales sean la única cosa nueva y significativa, como si antes no se hubiese hecho nada; por otra parte, a perder la masa crítica y, añadiría, la humildad necesaria y la correcta actitud científica, por lo que una mirada desapasionada del pasado es necesaria para trabajar el presente. En algunos aspectos, las investigaciones sobre los niños y la televisión (y no solo éstas) se suceden unas a otras como un objeto de moda: sobre la última en orden cronológico se presta una gran atención, sobre todo si propone "algo nuevo"; una atención que se pierde en poco tiempo revelando su carácter efímero.

Si hoy leemos o escuchamos a alguien afirmar que "la televisión es una niñera económica y siempre a disposición”, nos parece una afirmación basada en una crítica pedagógica sobre el medio; pero nos quedamos estupefactos, aunque solo por un momento, si descubrimos que es una de las consideraciones que podemos leer en la investigación de Ira Glick y Sidney Levi, Living with Televisión, publicada en 1962 en Chicago. Su investigación, que se mueve en parámetros esencialmente cualitativos, tiene su núcleo en identificar el público televisivo no como una masa indistinta de sujetos, sino como una realidad compleja cuyas actitudes, comportamientos, ideologías hacia el medio dependen fuertemente de la edad y del género, de las condiciones sociales y culturales entrecruzadas entre ellas, pero que tienen en la familia el punto de referencia en el que se determinan los "estilos" dominantes al relacionarse con la televisión.

Los investigadores llegan así a identificar tres tipos de público, que describen a través de la composición de aquellas variables: aquellos que "aceptan la televisión incondicionalmente", el público de los "contestatarios de la televisión", finalmente aquellos que manifiestan una "actitud acomodaticia". Las tres definiciones son per sé bastante elocuentes. En cada uno de estos grupos de público se manifiestan elecciones y orientaciones que asumen también claras valencias educativas en el ámbito de la pedagogía de la familia. Por supuesto, se trata de categorizaciones que podrían parecer un poco abstractas o rígidas y nos sugerirían, implícitamente, que en la realidad es más verosímil que haya conjuntos de actitudes diversas en sujetos diversos según las situaciones; o que, más correctamente, se debería hablar de "modelos prevalentes" que no excluyen otras categorías intermedias, donde es posible una cierta ambigüedad de comportamientos. 
No hay duda, en cualquier caso, de que el modelo epistemológico de reducir la complejidad a categorías claras y distintas ordenadas por taxonomías, sobre las que la ciencia occidental ha construido gran parte de su cimiento metodológico y gnoseológico, ha caracterizado también el campo de la investigación del que nos ocupamos. La televisión, no como hardware sino como software y como tool, ha sido fuertemente objetivada, tratada por lo tanto como si fuera una materia observable, describible y medible sobre la base de parámetros y definiciones asertivas.

Releyendo la investigación de Glick y Levy y la descripción que ellos hacen de los "diversos tipos de público", he pensado inmediatamente en Umberto Eco y en la definición de "apocalípticos e integrados" que da título a uno de sus libros más famosos, aparecido en 1964. Convertido después en una suerte de paradigma o de clave de lectura citada y utilizada en muchos contextos, Apocalípticos en integrados expresa una visión rígida y radical al describir el modo en el que los sujetos se relacionan con los mass media. Él ni siquiera se plantea la hipótesis de que pueda haber una categoría intermedia, aquella que Glick y Levy definen como "actitud acomodaticia". La de Umberto Eco es una tesis que no se basa en la investigación empírica, sino que desarrolla una teoría crítica de la cultura, y como tal está dotada de una excepcional fuerza retórica e icástica, parangonable a las de otros conceptos-llave sobre los que se ha construido una especie de mapa teórico sobre la cultura de los medios. Basta pensar en conceptos como "industria cultural" de Horkheimer y Adorno (1947), "el medio es el mensaje" de McLuhan (1966), la "escuela paralela" de Friedmann (1966), hasta la "desaparición de la infancia" de Postman (1982) y las visones inquietantes de Mary Winn (la droga televisiva), y de Condry y Popper (la televisión, mala maestra, 1994).

\section{Los mundos paralelos de la investigación y de la televisión}

Parece que la investigación no tiene sobre la televisión ningún efecto equiparable a lo que ocurre en otros campos de la vida social, donde la investigación desempeña una importante función de desarrollo y de cambio. El proceso evolutivo de la televisión en la sociedad y su éxito casi no tienen relación con la enorme masa de investigaciones que se producen en el mundo sobre este medio desde que apareció hace más o menos 60 años. Si se excluyen las indagaciones estrictamente relacionadas con la "investigación de mercado", los índices de audiencia y de preferencias, el comportamiento del público en cuanto a la relación audiencia-consumo, las investigaciones psicológicas, sociológicas, pedagógicas, con la enorme cantidad de datos y de teorías que han producido, no han influido en la elección de políticas culturales ni en los modelos creativos y productivos de la televisión. Los dos mundos, el de la investigación sobre la televisión y el de la producción de televisión han ido siempre cada uno por su cuenta, mirándose recíprocamente desde la distancia. 
La excepción se produce con algunos programas nacidos con un evidente carácter experimental o como intentos educativos, y por eso financiados desde el principio o periódicamente por actividades de investigación capaces de hacer dialogar a los autores con los productores televisivos. El ejemplo más famoso es Barrio Sésamo (Sesame Street), el programa de la CTW (Childrem Television Workshop), nacido en 1969 y después difundido y adaptado en otros 60 países del mundo. En las intenciones de su creadora, Johan Cooney, se trataba de un programa educativo experimental dirigido a niños en edad preescolar. Para valorar su eficacia era por lo tanto necesario acompañar la programación con una instalación de investigación capaz de suministrar continuos feed-back sobre su eficacia comunicativa y formativa, tales que permitieran ajustes e innovaciones.

Una específica División de Investigación interna a la CTW trabajaba en esta dirección, pero al mismo tiempo el programa estimulaba otras investigaciones externas al circuito productivo y en todos los países donde Barrio Sésamo era propuesto al público infantil (Lesser, 1974). Haciendo una indagación somera en algunas bases de datos significativas, se pueden contar más de 400 títulos de investigación empírica y experimental realizadas sobre este programa, o sobre la relación televisión/educación donde de algún modo se hace referencia a Barrio Sésamo. Obviamente, se trata de trabajos de investigación que en parte enfatizan los aspectos positivos del programa, pero por otra parte revelan consideraciones críticas. Estamos hablando en cualquier caso de un programa de éxito y de un gran negocio en el mercado del eduteinment que ha implicado y sigue implicando millones de niños en el mundo: un verdadero fenómeno global de la televisión educativa, al que la investigación ha proporcionado una efectiva posibilidad de desarrollo.

En Italia puedo recordar dos ejemplos recientes, obviamente de alcance mucho menor, pero significativo. El primero, en el que yo mismo he participado, se refiere al programa L'Albero azzurro [El árbol azul], nacido en 1990 y dedicado a un público de niños de entre 3 y 6 años. La RAI, que producía y emitía el programa, encargó a un equipo del Departamento de Ciencias de la Educación de la Universidad de Bolonia acompañar los tres primeros años del programa con planes de investigación cuyos temas se decidían de común acuerdo entre las dos instituciones; los reportes eran objeto de encuentros periódicos entre los autores, los productores y los investigadores. Durante la década de los 90 L'Albero azzurro en Italia se había convertido en una suerte de emblema de la televisión de calidad para la infancia (Farné, Gherardi, 1994; Farné, 1997).

El otro ejemplo es Melevisione, también dirigido a la franja de edad preescolar. Nacido en 1999 a la estela de L'Albero azzurro, del que hereda varios autores, también Melevisione promueve un plan de estudios y de investigaciones en esta ocasión con un equipo de la Universidad de Turín (Coggi, 2003, 2004). La característica de ambos programas es dar forma a un lenguaje y una comunicación televisiva distintos de aquellos 
que caracterizan las retransmisiones de más largo recorrido dirigidas al público infantil, asumiendo una intención claramente educativa y sugestiva, que requiere inversión en nuevas formas de comunicación y dramaturgia televisiva dirigidas a la infancia.

En estos dos ejemplos tomados de la televisión pública italiana, como en aquel más famoso de Barrio Sésamo, la unión entre la televisión y la investigación no se produce por casualidad en programas para la infancia, donde la explícita intencionalidad educativa no quiere renunciar a la especificidad del formato televisivo. En este sentido, la investigación se convierte en un socio fundamental de la televisión, y el concepto (el estereotipo) de que la televisión es dañina para los niños, sustentado por una cantidad de análisis y de investigaciones que durante años han alimentado la gran tensión emotiva sobre este tema, podría volverse del revés afirmando que los niños son buenos para la televisión. Cuando son vistos y tratados como un público especial e importante, los niños representan un reto comunicativo que obliga a la televisión a hacer un esfuerzo verdaderamente importante.

Dejando de lado algunas excepciones, como se ha dicho, nos encontramos de frente con una radical dicotomía entre quienes hacen la televisión y quienes investigan sobre la televisión. En el primer caso, los autores y los productores televisivos miran la investigación científica y crítica con distancia y superficialidad: se trata de discursos, opiniones, provocaciones que pueden suscitar un interés momentáneo, protagonizados por una elite intelectual y por lo tanto sin ninguna repercusión sobre el trabajo de quienes hacen la televisión. Hace algunos años un productor televisivo, en un debate en el que yo participaba, dijo, con un tono entre irónico y sarcástico, que muchos intelectuales deberían estar agradecidos a la televisión por cómo es, más que nada porque les ofrece un formidable campo de estudios y de investigaciones...

Por otra parte, quien investiga sobre la televisión generalmente no conoce el interior de la realidad televisiva, sino que la mira desde fuera, con frecuencia desde una actitud preconcebidamente crítica. He llegado a conocer a investigadores de este campo que declaran abiertamente no solo su aversión hacia el network y el broadcasting, sino incluso que no ven la televisión, y sobre todo los programas para niños, que consideran especialmente desagradables. Es la actitud intelectual de quien se siente en disposición de emitir un juicio con un valor de "cientificidad" sin plantearse el problema de qué significa "conocimiento" del objeto de investigación. No pretendo con esto sostener que las investigaciones sobre los niños y la televisión sean en gran medida pre-concebidas, sino más bien que con frecuencia se resienten de una actitud "autocentrada", de un enfoque demasiado bajo respecto al propio "objeto".

En esta radical separación entre quien hace la televisión y quien investiga sobre la televisión encontramos una posible explicación de nuestra incomodidad respecto al tema de la infancia concebida como "público 
televisivo", sobre la base de dos aspectos. El primero es la baja consideración de este público y su marginalidad en el marco de las inversiones ideativas y productivas de las network. La experimentación y la innovación en los programas para la infancia son bajísimas frente al dato de que los niños constituyen un importante target desde el punto de vista de las mercancías y los productos de consumo vehiculados a través de la televisión.

Desde el punto de vista del mercado publicitario, este público representa para las televisiones (sobre todo las comerciales, aunque también las públicas) un sector que garantiza "la massima resa con la minima spesa” (el máximo beneficio con la mínima inversión). Durante el periodo en que trabajaba en la investigación del programa L'Albero azzurro recuerdo que un funcionario de la RAI dijo en una reunión que un año entero de programación de L'Albero azzurro (incluidos los gastos de la investigación) costaba como ¡una sola emisión del show del sábado noche! Sería interesante saber por parte de un network televisivo comercial de gran difusión cuál es la relación entre lo que ingresa con la cuenta de la publicidad de todos los productos directamente dirigidos a la infancia y lo que invierte en producir retransmisiones para este público. Si nos referimos a la televisión pública, la misma pregunta debería incluir también la parte de financiación con dinero público que el network recibe a través de la tasa que los ciudadanos pagan, como ocurre en Italia.

El segundo aspecto se refiere a la investigación. Tenemos a nuestra disposición medio siglo de investigación empírica, experimental, crítica sobre la relación niños-televisión, que ha implicado a las ciencias sociales, de la educación, de la comunicación, de la salud. En nuestra historia jamás había ocurrido que un medio generase tal cantidad de estudios y de investigaciones, hasta tal punto que es prácticamente imposible abarcarlo por completo por parte de quien hoy continúa investigando este campo. Pero deberíamos preguntarnos cuántas y cuáles son las consideraciones científicas que surgen de las mismas investigaciones y que constituyen piedras angulares, principios adquiridos y seguros. Se trata, quizá, de aceptar abiertamente y sin prejuicios la fragilidad de todo esto, la inconsistencia de los "datos de verdad", como la ciencia moderna querría llamarlos, y con todas las cautelas con que este concepto es usado en el ámbito de las ciencias humanas.

Por una parte deberíamos reconocer que un gran número de investigaciones sobre los niños y la televisión son en gran medida parodias de sí mismas, variaciones sobre el tema... Por otra, que aquéllas, volviendo sobre temas y problemas con técnicas y métodos de investigación cuantitativos o cualitativos, en el buscar y rebuscar confirmaciones y refutaciones sobre cuestiones destinadas a permanecer constantemente abiertas, tienen el mérito de afirmar el valor de aquel “sentido común” que da Vico a Gádamer (1960) constituye 
a estas alturas un punto esencial de la hermenéutica epistemológica sobre el sentido del "hacer ciencia" para el hombre moderno. Aquí no se trata de asumir una actitud escéptica o nihilista sobre las posibilidades (y la necesidad) de la investigación, sino de recuperar una "vuelta a las cosas mismas", para decirlo con el lenguaje de la fenomenología, preguntándose qué significa esto cuando se habla, por ejemplo, de la "televisión como experiencia infantil". Y ¿qué es si no "sentido común", en el sentido más hondo de la ciencia-conocimiento (scienza/conoscenza) humana, el hecho de que de estas investigaciones emerjan y surjan consideraciones como la que he citado al principio y que sintetizan la investigación de Schramm, Lyle y Parcker?

Piénsese en los efectos de la violencia televisiva sobre el público infantil, en las que existen tesis diferentes sobre la base de varias investigaciones todas rigurosamente "científicas". Muchas de éstas no hacen sino evidenciar la centralidad y el problematismo de los sistemas de vida (cotidiana, social, educativa...) respecto a cada determinismo rígido. Si se toman las investigaciones que durante años han tratado algunos de los típicos problemas vinculados con la relación niños-televisión, como por ejemplo la influencia que puede tener el medio sobre el rendimiento escolar, sobre la inactividad física, sobre la socialización, sobre modelos culturales, alimenticios, etc., se descubre que, en gran medida, los resultados científicamente relevantes están todos en un puñado de afirmaciones ampliamente compartibles sobre la base de un "sentido común", al cual la investigación no hace sino aportar elementos importantes de confirmación y de tranquilidad.

Que sea necesario volver a pensar y definir el sentido (o el sinsentido) de la investigación en este campo nos lo sugieren también otras consideraciones. Entre el mundo de la investigación sobre la televisión y el mundo de las televisiones no se ha creado un circuito virtuoso, si se excluyen algunos casos significativos y esporádicos, de los cuales en parte se ha hablado. Las televisiones han continuado pensando en sí mismas decenios tras decenios en términos de optimización de los beneficios y de las audiencias, asumiendo hacia el público infantil un perfil bajo de tutela, lo que podríamos definir como actitud "negativa”. Esto significa en la práctica, garantizar los horarios de protección reforzada en los cuales no se insertan programas o publicidad con determinados contenidos de carácter violento, erótico, etc.; indicar con una específica señal o con avisos los programas vespertinos de los que se desaconseja la visión de los niños o de los que se aconseja que junto con ellos esté un adulto, y así ir tirando sobre la base de normas en parte dictadas por una legislación hecha a medida, en parte por códigos de autorregulación (alguien prefiere hablar también de "autocensura”). Sin quitar valor a estas medidas de intervención, hay que reconocer todos sus límites: ¿quién determina los criterios de selección y de señalación de los programas? ¿Existen investigaciones periódicas que documentan su eficacia? ¿Qué sistemas de "valores" en términos pedagógicos y culturales conforman el marco para un sistema de control? 
Es la actitud positiva el verdadero punto débil, o sea, lo que tendría que elaborar ideas y proyectos de televisión para niños, sean de ficción o de no ficción, experimentar modalidades comunicativas nuevas, crear sinergias con otros ámbitos de la cultura para la infancia. Bastaría considerar los desarrollos ideativos y productivos que en los últimos treinta años han caracterizado sectores como el teatro para niños y la literatura para la infancia, para darse cuenta de qué poco significativa es la televisión para este público en términos de innovación. Esto a pesar de que la televisión dispone de recursos económicos enormes respecto a los del teatro y la industria editorial.

Ante una televisión sustancialmente insensible o indiferente hacia la investigación, la misma investigación ha terminado dando rodeos inútiles o dando lugar a soluciones que no iban más allá de la estrecha elite de los especialistas del sector, en una especie de cortocircuito científico. Algo parecido, por ciertos sectores, a lo que pasa en la escuela, donde si se tuviera que medir los efectos de la investigación psicopedagógica y didáctica en términos de innovación sobre modelos de enseñanza-aprendizaje y de gestión escolar, tendríamos objetivamente que reconocer que la escuela está parada, o se mueve con una velocidad tan baja que resulta imperceptible al ojo humano. Aquí también podemos decir que la escuela y la investigación no están en "relación recíproca"; el mundo escolar, autocentrado en el propio trabajo cotidiano y en el propio aparato institucional, cree no necesitar la contribución externa de la investigación, de "su punto de vista”, y ésta cree no necesitar un interlocutor como la escuela, limitándose a contestar solo a sus propias instancias de "libertad de investigación".

Por lo tanto, la pregunta es si resulta realista esperar que la investigación sobre la relación entre niños/televisión produzca cambios en la realidad televisiva y sus contextos de vida de la infancia, y qué procesos se deberían alimentar para que esto ocurra.

Puesto que las características negativas de una cierta programación televisiva ofrecida al público infantil (teniendo en cuenta obviamente todas las variables del caso: edad, estatus social, etc...) han sido ampliamente reveladas por la investigación, es legítimo preguntarse por qué la televisión no mejora la calidad de sus programas. La respuesta puede ser doble: o porque no es verdad que medio siglo de investigación haya producido conclusiones científicamente ciertas en lo referente a los efectos sobre la infancia, o porque el mundo de la televisión no puede permitirse tener en cuenta el mundo de la investigación científica en estos temas. O, tal vez, por ambas razones.

Ocurre lo mismo, más o menos, en la escuela. Pongo un ejemplo: todos los estudios y las experimentaciones sobre métodos activos que confirmarían la eficacia pedagógica y didáctica no han logrado una difusión a gran escala, y el modelo de enseñanza/aprendizaje de tipo transmisivo sigue ampliamente practicado. 
Asumiendo el punto de vista científico, debemos afirmar que si las cosas están así, entonces no es verdad que las metodologías activas sean didácticamente más eficaces que las transmisivas, de lo contrario habrían entrado a formar parte de la didáctica escolar habitual. O bien que la escuela, fisiológicamente, o sea, por la propia "naturaleza institucional" no es capaz de asumir de una vez los resultados de la investigación psicopedagógica.

No debe parecer anómalo el paralelismo entre la televisión y la escuela respecto a este tema. En la especificidad y en la diversidad ambas instituciones se basan en la comunicación de mensajes y la relación diaria con un gran público sobre el que se ejercita una significativa influencia (condicionamiento) cultural; ambas elaboran "programas" basados en la continuidad y en la asistencia asidua. En el caso específico de la historia italiana contemporánea, además, quiero recordar que la televisión ha desarrollado en el arco de 15 años, hasta el final de los años sesenta, un rol pedagógico fundamental en la lucha contra el analfabetismo, en la difusión de la lengua italiana y en la divulgación cultural y científica en todos los campos (Farné, 1993).

No se produce aquí lo que en otros ámbitos de la sociedad: un circuito virtuoso (aunque problemático y no siempre lineal...) entre la investigación y la innovación. Pensemos en la medicina, o en sectores como el de las tecnologías, la energía, etc. ¿Deberíamos concluir, tal vez, que en general la investigación sobre la televisión y los niños es una investigación "improductiva"? No lo creo. Más bien deberíamos reflexionar sobre el hecho de que, a diferencia de otros sectores, la investigación en este campo generalmente no cuenta con las televisiones como socios que contribuyan a su financiación. Se puede entender esto como una garantía para la "libertad de la investigación" respecto a quienes la encargan y financian, que podrían pretender condicionarla. Más en general, una situación que permite a ambos, quien hace la investigación y quien hace la televisión, tener las manos libres en el propio territorio y no tener que enfrentarse al otro.

Me viene a la mente un aforismo de Groucho Marx que decía: "Encuentro la televisión un medio muy educativo: cada vez que alguien la enciende me voy a otra habitación a leer un libro".

\section{Bibliografía}

Coggi C. (ed.), (2003): Valutare la TV per i bambini: vie alla qualità e all'uso educativo, Franco Angeli, Milán.

Coggi C. (ed.), (2004): Migliorare la qualità della TV per bambini, Franco Angeli, Milán.

Eco U. (1964): Apocalittici e Integrati, Bompiani, Milán.

Farné R. (1987): “L'Albero azzurro nella prescuola: alcune note di ricerca”, Ikon, 34, pp.109-145.

Farné R. (1993): Buona maestra TV. La RAI e l'educazione da "Non è mai troppo tardi" a "Quark", Carocci, Roma. 
Farné R., GHERARDIV. (1994): All'ombra di un Albero azzurro. Ricerca su un programma televisivo per bambini, CLUEB, Bolonia.

Friedmann G. (1966): “La scuola parallela”, Il Mulino, 7-8, pp. 677-691.

Gadamer H.G. (1960): Wahrheit und Methode. Grundzüge der philosophischen Hermeneutik, Tübingen, (trad. it., 1972: Verità e metodo. Lineamenti di un'ermeneutica filosofica, Bompiani, Milán).

Glick I.O., LEVY S.J. (1962): Living with Television, Aldine Puplishing Company, Chicago, (trad. it., 1975: Vivere con la televisione, Franco Angeli, Milán).

Horkheimer M., ADORNO T.W. (1947): Dialektik der Aufklärung, Philosophische Fragmente, Querido Verlag, Amsterdam, (trad. it. 1966: Dialettica dell'Illuminismo, Einaudi, Turín).

Lesser G.S. (1974): Children and Television. Lessons from Sesame Street, Random House, Nueva York.

McLuhan M. (1964): Understanding Media, Mc Graw-Hill Book Company, NuevaYork, (trad. it., 1967: Gli strumenti del comunicare, Il Saggiatore, Milán).

Popper K.R. (1994): “Una patente per fare TV”, en Popper K.R., Con dry J., Cattiva maestra televisione, Reset, Milán.

Postman N. (1982): The Disappearence of Childhood, Delacorte Press, Nueva York, (trad. it., 1984: La scomparsa dell'infanzia. Ecologia delle età della vita, Armando, Roma).

Schramm W., LYLE J., PARKER E. (1961): Television in the Lives of our children, Board of the Trustees of the Leland Stanford Junior University Press, Stanford California, (trad. it. 1971: La televisione nella vita dei nostri figli, Franco Angeli, Milán).

Winn M. (1979): Unplugging the Plug-in Drug, Weking, Nueva York, (trad. it., 1983, La droga televisiva, Armando, Roma).

$24\left|n^{\circ} 7\right|$ doxa.comunicación 